\title{
Wireless charing pillow for a fully implantable hearing aid: Design of a circular array coil based on finite element analysis for reducing magnetic weak zones
}

\author{
Hyung-Gyu Lim ${ }^{\mathrm{a}}$, Jong Hoon Kim ${ }^{\mathrm{b}}$, Dong Ho Shin ${ }^{\mathrm{a}}$, Seong Tak Woo ${ }^{\mathrm{a}}$, Ki Woong Seong ${ }^{\mathrm{c}}$, \\ Jyung Hyun Lee ${ }^{\mathrm{c}}$, Myoung Nam Kim ${ }^{\mathrm{d}}$, Qun Wei ${ }^{\mathrm{e}}$ and Jin-Ho Cho ${ }^{\mathrm{a},{ }^{*}}$ \\ ${ }^{a}$ Graduate School of Electronics Engineering, Kyungpook National University, 80 Daehak-ro, Buk-gu, \\ Daegu 702-701, Korea \\ ${ }^{b}$ Department of Medical and Biological Engineering, Graduate School, Kyungpook National \\ University, 680 Gukchaebosang-ro, Jung-gu, Daegu 700-422, Korea \\ ${ }^{c}$ Department of Biomedical Engineering, Kyungpook National University Hospital, 130 Dongdeok-ro, \\ Jung-gu, Daegu 700-721, Korea \\ ${ }^{d}$ Department of Biomedical Engineering, School of Medicine, Kyungpook National University, 680 \\ Gukchaebosang-ro, Jung-gu, Daegu 700-422, Korea \\ ${ }^{e}$ Department of Biomedical Engineering, School of Medicine, Keimyung University, 1095 Dalgubeol- \\ daero, Dalseo-gu, Daegu 704-701, Korea
}

\begin{abstract}
Many types of fully implantable hearing aids have been developed. Most of these devices are implanted behind the ear. To maintain the implanted device for a long period of time, a rechargeable battery and wireless power transmission are used. Because inductive coupling is the most renowned method for wireless power transmission, many types of fully implantable hearing aids are transcutaneously powered using inductively coupled coils. Some patients with an implantable hearing aid require a method for conveniently charging their hearing aid while they are resting or sleeping. To address this need, a wireless charging pillow has been developed that employs a circular array coil as one of its primary parts. In this device, all primary coils are simultaneously driven to maintain an effective charging area regardless of head motion. In this case, however, there may be a magnetic weak zone that cannot be charged at the specific secondary coil's location on the array coil. In this study, assuming that a maximum charging distance is $4 \mathrm{~cm}$, a circular array coil—serving as a primary part of the charging pillow - was designed using finite element analysis. Based on experimental results, the proposed device can charge an implantable hearing aid without a magnetic weak zone within $4 \mathrm{~cm}$ of the perpendicular distance between the primary and secondary coils.
\end{abstract}

Keywords: Wireless charging pillow, fully implantable hearing aid, inductive coupling, circular array coil, magnetic weak zone

\footnotetext{
* Address for correspondence: Jin-Ho Cho, Graduate School of Electronics Engineering, Kyungpook National University, 80 Daehak-ro, Buk-gu, Daegu 702-701, Korea. Tel.: +82-53-427-5538; Fax: +82-53-427-5539; E-mail: jhcho@ee.knu.ac.kr.
}

0959-2989/15/\$35.00 @ 2015 - IOS Press and the authors.

This article is published with Open Access and distributed under the terms of the Creative Commons Attribution and Non-Commercial License. 


\section{Introduction}

Many people throughout the world suffer from hearing loss. Although many kinds of airconductional hearing aids have been conventionally used, patients have often been dissatisfied with them on account of several problems, such as 'howling' sounds, occlusion, social stigma, and so on [1, 2]. To address such limitations, studies of implantable hearing aids have been undertaken. Recent research trends have focused on a full implant with a microphone and battery [3]. Most fully implantable hearing aids are completely implanted beneath the skin of the temporal bone except for the output transducer $[4,5]$. Thus, wireless power transmission is required for periodic battery charging. Inductive coupling with two coils that are transcutaneously coupled has been typically used for the wireless power transmission $[6,7]$.

Some patients with a fully implantable hearing aid require an additional instrument for charging while the patient rests. Thus, wireless charging pillow has been developed with an array of circular coils. In this pillow, all circular coils should be simultaneously activated. This enables enhanced probability of inductive coupling with a secondary coil regardless of head movement. At any boundary area between the circular coils, however, there may sometimes be a magnetic weak zone that cannot be effectively charged even though the secondary coil is set upon the circular array coil.

In this paper, we propose for the wireless charging pillow a circular array coil that improves the wireless charging efficiency by reducing the magnetic weak zone. Considering an existing secondary coil dimension [8], the size of each circular coil of the array was determined after executing wireless power transmission analysis using the finite element method. An array coil with nine circular coils (3 $\times 3$ ), a secondary coil, and electronic circuits were implemented. We then performed a wireless charging experiment for best and worst cases. To verify the feasibility of the array coils, resultant data were compared and analyzed.

\section{Fully implantable hearing aid and wireless charging pillow in Korea}

The fully implantable hearing aid has been studied to overcome several limitations of conventional hearing aids. In Korea, a type of fully implantable hearing aid with an electromagnetic transducer has been developed. This device includes an implantable microphone, a vibrating transducer, and a main system with processing circuits and a rechargeable battery. As shown in Figure 1, the fully implantable hearing aid is surgically implanted at the temporal bone for long-term use. A transcutaneous method is employed for charging to prevent infection.

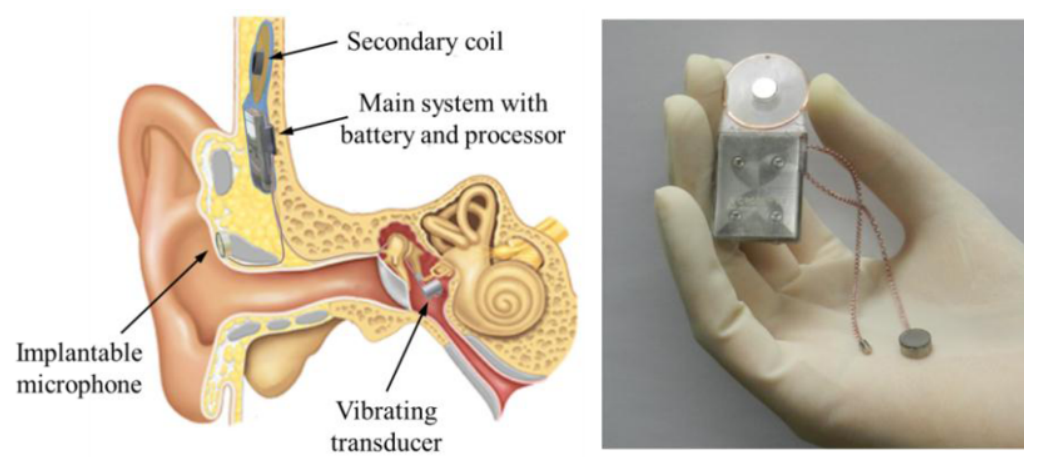

Fig. 1. Schematic and prototype of a fully implantable hearing aid developed in Korea. 
The proposed wireless charging pillow is based on inductive coupling between coils. A circular array with plural coils, instead of a single primary coil, is used regardless of head movement. Each coil is connected with combined capacitors for LC parallel resonance at $134.2 \mathrm{kHz}$. A block diagram of the proposed device, except for the display and protecting circuit, is shown in Figure 2. All coils of the array are uniformly arranged on a flat plane and are simultaneously driven by the same phase current, as shown in Figure 3(a). When the device is being operated, induced voltage is generated by inductive coupling between the circular array coil and secondary coil. The rectified voltage is supplied to a battery charger chip that charges the battery. However, if the secondary coil is located in a magnetic weak zone (e.g., the boundary area between the coils), as shown in Figure 3(b), the battery may not be capable of being charged. Therefore, a circular array coil of a reasonable size is required.

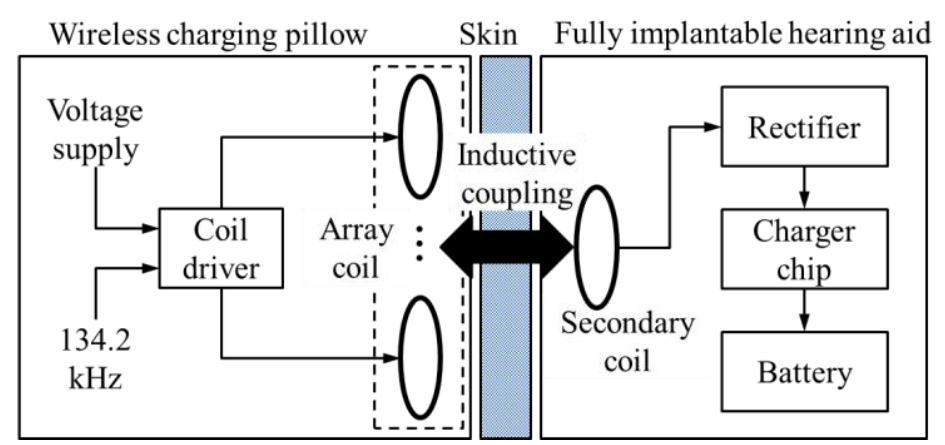

Fig. 2. Block diagram of the proposed wireless charging pillow and power receiving circuits in a fully implantable hearing aid.
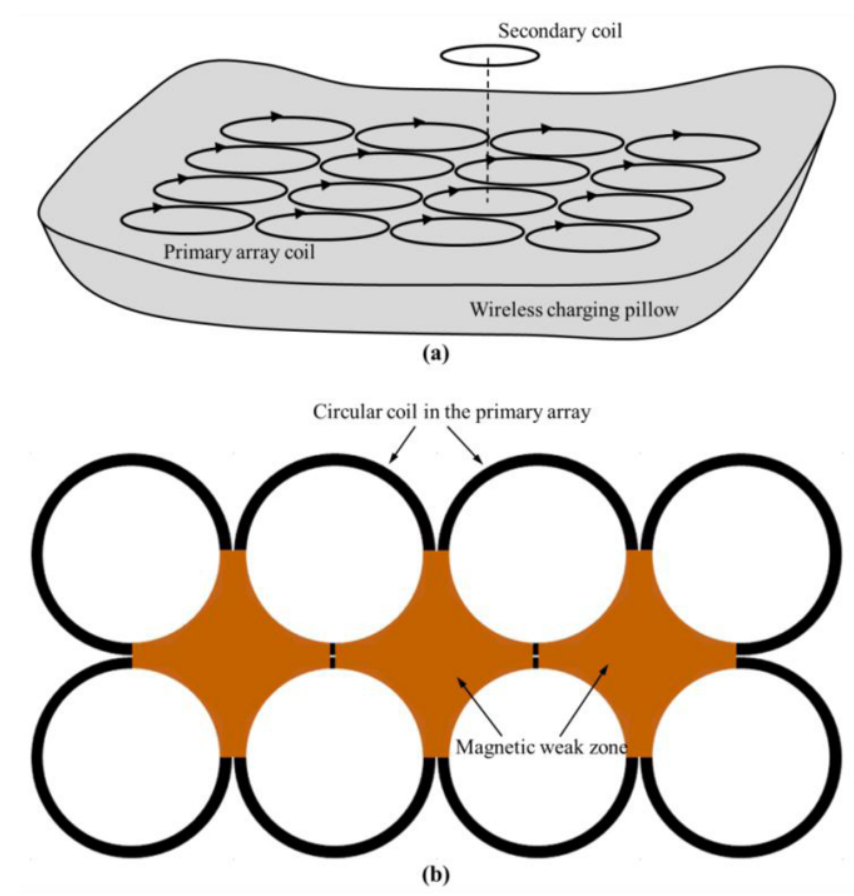

Fig. 3. Wireless charging pillow with a circular array coil: (a) schematic view, and (b) periodically distributed magnetic weak zones (top view). It was predicted that battery charging in the area would be difficult. 


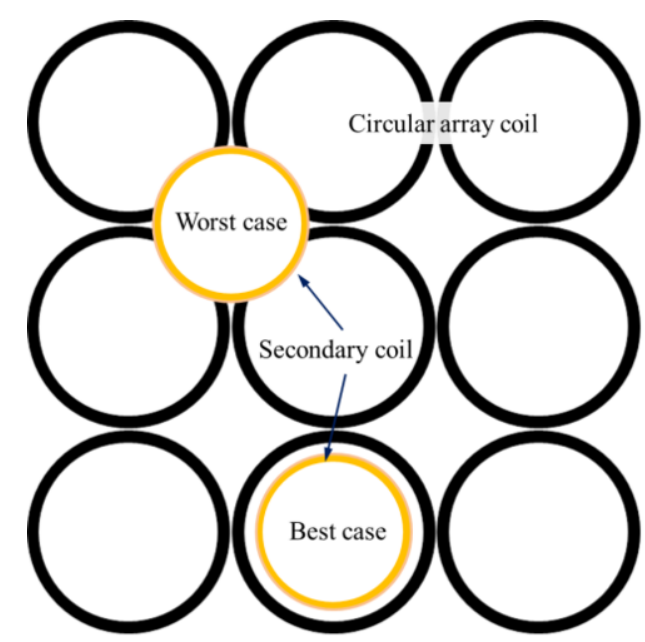

Fig. 4. Considerations for the simulation of wireless power transmission: the best and worst cases of the secondary coil on the circular array coil.

Table 1

Specifications and dimension of the coils

\begin{tabular}{lll}
\hline & Each circular coil in the array & Secondary coil \\
\hline Inner diameter & Variable & $26 \mathrm{~mm}$ \\
Outer diameter & Inner diameter $+4 \mathrm{~mm}$ & $28 \mathrm{~mm}$ \\
Height & $2 \mathrm{~mm}$ & $1 \mathrm{~mm}$ \\
Coil thickness & $0.25 \mathrm{~mm}$ & $0.1 \mathrm{~mm}$ \\
Turns & 36 & 85 \\
Material & Copper & Copper \\
Inductance & Variable & $420 \mu \mathrm{H}$ \\
Peak current & $1 \mathrm{~A}$ & \\
\hline
\end{tabular}

\section{Circular array coil design}

As mentioned above, a magnetic weak zone can be periodically distributed. To design a circular array coil that can reduce magnetic weak zones, several situations by head movement were considered for the charging process. Finally, as shown in Figure 4, the best and worst cases were assumed for analysis of power transmission using a finite element method. Moreover, the array size $(3 \times 3)$ was limited for reducing the computational time. This analysis was performed using Magnetostatic Analysis System in ANSYS Workbench (ANSYS, Inc.). Table 1 shows the specifications of the primary circular coil and secondary coil. In the simulation, after the secondary coil dimension was fixed, each interlinked magnetic flux density at the secondary coil was calculated in accordance with the varying perpendicular distance between the array and secondary coil and each coil's diameter of the array, respectively.

Figure 5 presents the simulated results. In the plot, red and blue lines denote the worst and best cases, respectively. The circular coil diameter was decided after the differences between the two results were calculated. To maintain overall uniformity of the magnetic field in the wireless charging pillow, the difference had to be minimized. Therefore, the outer diameter, inner diameter, and height dimensions of each circular coil were represented as $36 \mathrm{~mm}, 32 \mathrm{~mm}$, and $2 \mathrm{~mm}$. 

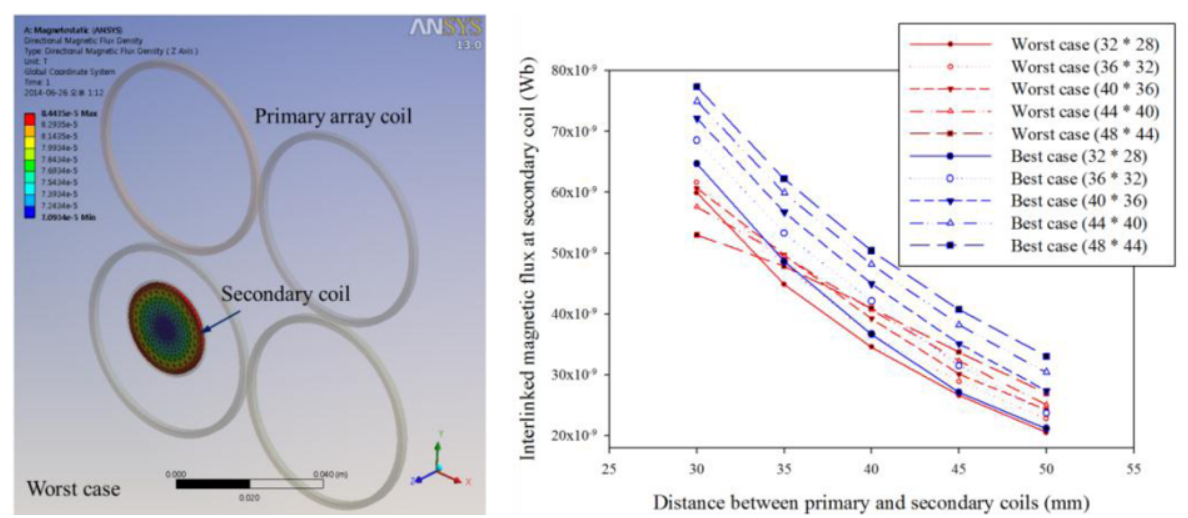

Fig. 5. Simulated results of interlinked magnetic flux density at the secondary coil versus the perpendicular distance between the coils, with (red line: worst case; blue line: best case).

\section{Experimental results}

To verify the charging feasibility of the designed array coil, the circular array coil $(3 \times 3)$, secondary coil, and all circuits were fabricated as a design result. The major components and specifications for the experiments are outlined in Table 2. At several test points, as shown in Figure 6(a), the induced voltage for the operating battery charger chip was measured by a digital multimeter (179, Fluke Co.). During the experiments listed in Figure 6(b), the perpendicular gap between the array and secondary coil was controlled by acrylic panels.

Table 2

Experimental materials and specifications

\begin{tabular}{lll}
\hline & Part number & Manufacturer \\
\hline Coil driver & SN754410 & Texas Instruments \\
Rectifier & BAT54S & Vishay \\
Charger chip & LTC4054L & Linear Technology \\
Li-ion battery & PD2032 & Korea Powercell \\
Operating frequency & $134.2 \mathrm{kHz}$ & \\
Supply voltage & $9 \mathrm{~V}$ and $12 \mathrm{~V}$ & \\
Battery capacity & $75 \mathrm{mAh}$ & \\
\hline
\end{tabular}
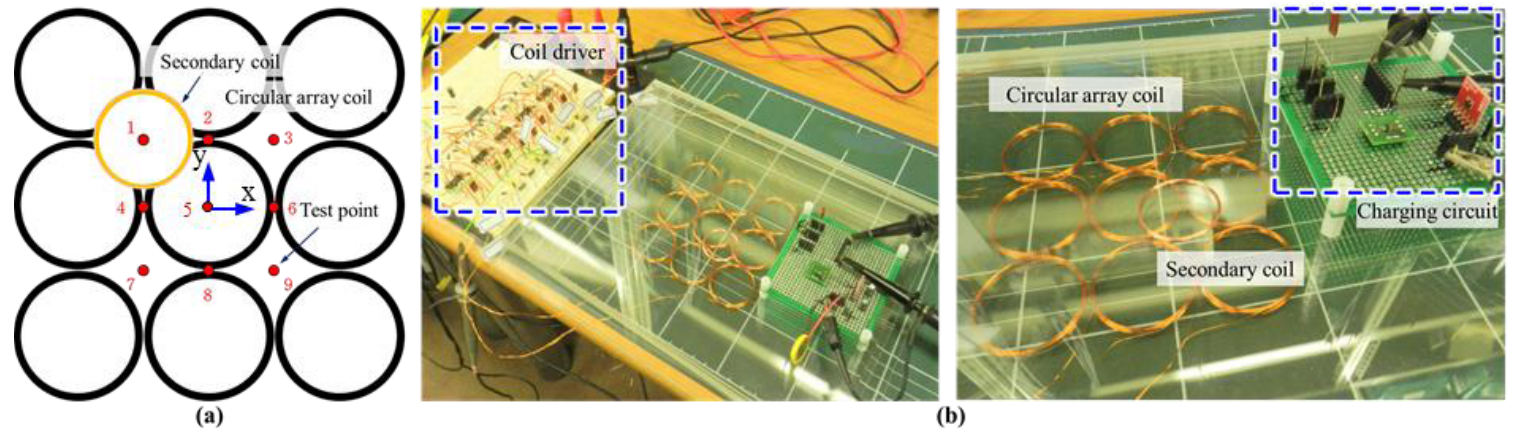

(b)

Fig. 6. Experiments of wireless power transmission using the fabricated circular coil array: (a) test points on the circular array coil (top view) and (b) experimental picture. 


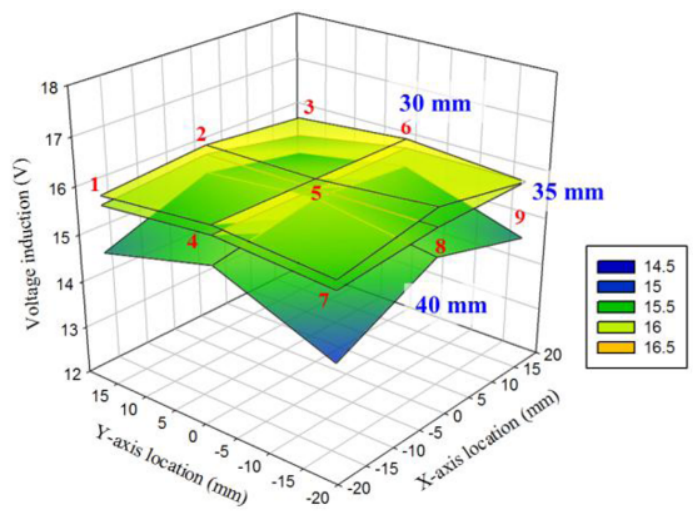

(a)

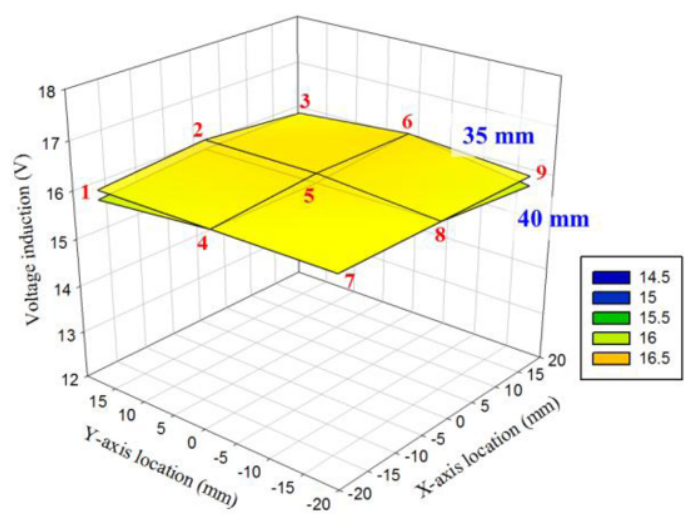

(b)

Fig. 7. Measurements of induced voltage according to varying supply voltage and distance between the array and secondary coil, respectively: (a) $9 \mathrm{~V}$ supply and (b) $12 \mathrm{~V}$ supply. Within $4 \mathrm{~cm}$ of the perpendicular distance, the wireless charging pillow maintains good charging performance.

Based on the results shown in Figure 7, the secondary coil was located at both best and worst positions; nevertheless, the battery could be easily charged within $4 \mathrm{~cm}$ distance since the all measurements was above the operating voltage of charger chip [9]. Further, the results in the best and worst cases were similar. Thus, the overall magnetic uniformity of the wireless charging pillow could be preserved.

\section{Conclusion}

Hearing impaired patients using a fully implantable hearing aid require additional equipment for charging the device battery without bodily constraints. Therefore, wireless charging pillow with a primary circular array coil was proposed in this paper. To overcome abnormal power transmission by head movement in sleep, the proposed array coil was designed to charge the hearing aid even if the device is located in a magnetic weak zone within $4 \mathrm{~cm}$ of the coil gap. According to the results of wireless powering experiments, the array coil's feasibility for the wireless charging pillow was verified.

At least, the pillow's size should not be less than the minimal dimensions (length: 300 and width: $200 \mathrm{~mm}$ ) with consideration of a normal head dimension. Hereafter, the number of coils in the array will be increased accordingly. By resolving an error of the rotational position of the secondary coil and by ensuring safety from the electromagnetic flux, the wireless charging pillow can be made much more convenient for patients.

\section{Acknowledgments}

This work was supported by the National Research Foundation of Korea (NRF) grants funded by the Korea government (No. 2013R1A1A2004991, No. 2013R1A2A1A09015677 and No. 2015R1A2 A2A03006113). 


\section{References}

[1] A.V. Hodges and T.J. Balkany, Cochlear implants for sensorineural hearing loss, Hospital Physician 38 (2002), 22-28.

[2] I. Todt, R.O. Seidl, M. Gross and A. Ernst, Comparison of different vibrant sound bridge audio processors with conventional hearing aids, Otology \& Neurotology 23 (2002), 669-673.

[3] D.S. Haynes, J.A. Young, G.B. Wanna and M.E. Glasscock III, Middle ear implantable hearing devices, Trends in Amplification 13 (2009), 206-214.

[4] E.M. Kraus, J.A. Shohet and P.J. Catalano, Envoy Esteem totally implantable hearing system: Phase 2 trial, 1-year hearing results, Otolaryngology-Head and Neck Surgery 145 (2011), 100-109.

[5] L. Bruschini, F. Forli, A. Santoro, P. Bruschini and S. Berrettini, Fully implantable otologics MET Carina ${ }^{\text {TM }}$ device for the treatment of sensorineural hearing loss, Preliminary surgical and clinical results, Acta Otorhinolaryngologica 29 (2009), 79-85.

[6] B. Lenaerts and R. Puers, Omnidirectional inductive powering for biomedical implants, Springer, Dordrecht, Netherlands, 2009

[7] G. Wang, W. Liu, M. Sivaprakasam and G.A. Kendir, Design and analysis of an adaptive transcutaneous power telemetry for biomedical implants, IEEE Transactions on Circuits and Systems 52 (2005), 2109-2117.

[8] S.T. Woo, G.H. Lee, E.S. Jung, H.G. Lim, K.W. Seong, J.H. Lee, M.N. Kim and J.H. Cho, Speech quality evaluation of subcutaneously implanted microphone using in vivo experiment, Bio-Medical Materials and Engineering 24 (2014), $3685-3691$.

[9] LTC4054L datasheet, Linear Technology's Website, available at: http://www.linear.com/product/LTC4054L-4.2, June 19th, 2015. 